\title{
miR-486-5p Serves as a Diagnostic Biomarker for Sepsis and Its Predictive Value for Clinical Outcomes
}

\author{
Baobin Sun \\ Shubin Guo \\ Emergency Medicine Clinical Research \\ Center, Beijing Chao-Yang Hospital, \\ Capital Medical University, Beijing Key \\ Laboratory of Cardiopulmonary Cerebral \\ Resuscitation, Beijing, 100020, People's \\ Republic of China
}

Background: As a molecular detection method, miRNA can quickly diagnose and prevent diseases, intervene in disease as early as possible, and reduce mortality. This study was to investigate the potential clinical diagnostic and predictive significance of miR-486-5p in sepsis and its correlation with inflammation and disease severity.

Methods: The serum miR-486-5p in 108 sepsis, 60 pneumonia-infected, and 101 healthy controls were detected by RT-qPCR. Spearman coefficient detects the correlation between serum miRNA and disease severity indicators (APACHE II, SOFA scores), and inflammation indicators (CRP, PCT), respectively. The diagnostic significance of miR-486-5p in sepsis was analyzed by the ROC curve. Kaplan-Meier estimator and Cox regression hazards analysis of the predictive significance of serum miR-486-5p in 28-day survival from sepsis.

Results: Serum miR-486-5p was increased in sepsis patients compared with healthy control and pneumonia-infected patients $(P<0.001)$. And increased serum miR-486-5p was positively associated with disease severity (SOFA score and APACHE II score) and inflammation (CRP and PCT). Serum miR-486-5p can not only identify sepsis patients from healthy controls $(\mathrm{AUC}=0.914)$ but also significantly distinguish sepsis patients from pneumoniainfected patients $(\mathrm{AUC}=0.814)$, showing good potential as a diagnostic biomarker for sepsis. In addition, serum miR-486-5p was an independent predictor of 28-day survival (logrank $P=0.012$ ), and patients with high levels of miR-486-5p had a poorer overall 28-day survival $(\mathrm{HR}=3.057,95 \% \mathrm{CI}=1.385-17.817, P=0.014)$.

Conclusion: miR-486-5p is a potential diagnostic biomarker for sepsis, and its high level is significantly correlated with the disease severity and inflammation. In addition, miR-486-5p were predictive risk factors for 28-day survival in sepsis patients.

Keywords: miR-486-5p, sepsis, diagnosis biomarker, predictive
Correspondence: Shubin Guo Emergency Medicine Clinical Research Center, Beijing Chao-Yang Hospital, Capital Medical University, Beijing Key Laboratory of Cardiopulmonary Cerebral Resuscitation, 8 South Workers Stadium Road, Chaoyang District, Beijing, 100020, People's Republic of China

Tel + 86- I0-8523I000

Email biansuidan27।@I63.com

\section{Introduction}

Sepsis is a primary reason for death in intensive care units (ICU) around the world. It refers to the dysregulation of the body's response caused by infection, which leads to life-threatening organ dysfunction. ${ }^{1}$ It is reported that 1.7 million new cases of sepsis occur each year, with 270,000 deaths. ${ }^{1}$ The mortality rate accounted for $20-50 \%$ of ICU death. ${ }^{2}$ Although antibiotic therapy, ventilator management, fluid resuscitation, and vasopressors have improved sepsis patients to some extent, the prognosis is still not ideal. ${ }^{3}$ In addition, its early diagnosis mainly relies on a series of laboratory, biological and microbiological tests such as symptoms, urine, and secretions, which may cause delays in diagnosis and treatment. ${ }^{4}$ Therefore, it is 
necessary to develop new sensitive and rapid biological diagnoses and predictive biomarkers for identification and early intervention to reduce morbidity and mortality.

As an important endogenous non-coding posttranscriptional regulator, microRNAs negatively regulated the target mRNA through base pairing. Abnormal expression of miRNAs is not only participated in the important biological functions of disease but also proposed as a diagnostic and predictive biomarker due to its conservation and stability. ${ }^{5}$ As the abnormal miR-1184, ${ }^{6}$ miR-22-3p, ${ }^{7}$ and miR- $452^{8}$ have been proved to be potential biomarkers of sepsis. Among many miRNAs, miR-486-5p is located on human chromosome $8 \mathrm{p} 11.21$ and can be used as a potential biomarker for sarcopenia in the elderly, ${ }^{9}$ endometrial cancer, ${ }^{10}$ and chronic myelogenous leukemia. ${ }^{11}$ What is more, inflammation is involved in the pathogenesis of sepsis, ${ }^{12}$ and miR-486$5 \mathrm{p}$ as an inflammation regulator has been confirmed in many diseases. Such as miR-486-5p regulates polycystic ovary syndrome by regulating inflammation..$^{13}$ miR-486-5p is elevated in acute lung injury, and reducing its level can inhibit lipopolysaccharide-induced pneumonia in mice. ${ }^{14} \mathrm{~A}$ recent study compared multiple miRNAs differentially expressed in 8 sepsis patients and 8 healthy individuals, and miR-486-5p was among the upregulated miRNAs. ${ }^{15}$ However, the clinical value of miR-486-5p in sepsis is lacking.

Based on the above research reports, this study aims to estimate the effects of miR-486-5p on the clinical diagnosis, severity, inflammation, and prediction of 28-day survival outcome in sepsis patients, to find a reliable potential diagnostic and preventive biomarker.

\section{Materials and Methods}

\section{Ethics Statement}

The current study is under the Declaration of Helsinki and approved by the Ethics Committee of Beijing Chao-Yang Hospital. This study was conducted under the informed consent of healthy subjects and patients or guardians (unconscious or even comatous on the day of admission). Patients with sepsis are treated as stated by the sepsis treatment guidelines (the surviving sepsis campaign guidelines) and the specific guidelines of the relevant committees. ${ }^{16}$

\section{Participant Enrollment}

Sepsis patients were admitted to the ICU of Beijing ChaoYang Hospital continuously between January 2016 and January 2019. Inclusion criteria for patients in the current research were: 1) patients diagnosed with sepsis according to the Third International Consensus Definition of Sepsis and Septic shock (Sepsis-3); ${ }^{17}$ 2) Age $\geq 18$ years; 3) Willing to participate in the study. Exclusion criteria: 1) Died within one day of admission; 2) Solid tumors or hematologic malignant tumors; 3 ) Complicated autoimmune disease; 4) Recently received immunosuppressive drug therapy; 5) Pregnant women or lactating period; 6) Chronic diseases such as cardiovascular and cerebrovascular disease, liver and kidney disease. One hundred and eight sepsis patients who met the requirements were included according to the above criteria. In addition, 60 pneumonia-infected patients who did not meet the sepsis criteria were included in the present research. Finally, 101 healthy subjects of matched age who underwent physical examination were selected as the healthy control, which was no history of tumor, no systemic inflammation, and the biochemical indicators were normal. The flowchart of patient recruitment was provided in the Supplementary Materials.

\section{Clinical Data and Sample Collection}

The subject's age, gender, body mass index (BMI), serum creatinine (Scr), white blood cells (WBC), C-reactive protein (CRP), procalcitonin (PCT), and other basic clinical information were recorded in Table 1. In addition, acute pathology and chronic health evaluation II (APACHE II) and sequential organ failure assessment (SOFA) scores were performed within 24 $\mathrm{h}$ of admission to the ICU to assess the severity of the patient's disease and the severity of organ failure, with higher scores indicating higher severity. According to the definition of sepsis-3, the SOFA score of sepsis patients was $\geq 2$. And the SOFA score was mainly based on the initial sepsis-associated organ failure assessment, ${ }^{18,19}$ which included the $\mathrm{PaO} 2 / \mathrm{FiO} 2$ ratio, Glasgow Coma Scale, mean arterial pressure, vasopressor requirements, serum creatinine, or urine output, and platelet count.

\section{Specimen Collection and Serum}

\section{Separation}

$5 \mathrm{~mL}$ of peripheral venous blood was collected when the admission. After remaining at room temperature, the upper serum was collected by centrifugation and stored at $-80^{\circ} \mathrm{C}$.

\section{RT-qPCR Reaction}

Total RNA molecules were isolated and extracted from serum samples by adding TRIzol, chloroform, isopropanol, and $75 \%$ ethanol. After its purity and quality were detected, 
Table I Comparison of the Baseline Data of Study Objects

\begin{tabular}{|c|c|c|c|}
\hline Parameters & Health Control $(n=10 I)$ & Pneumonia-Infected $(n=60)$ & Sepsis $(n=108)$ \\
\hline Age (year) & $65.56 \pm 8.04$ & $63.05 \pm 7.93$ & $63.86 \pm 6.59$ \\
\hline Gender (male/female) & $54 / 47$ & $32 / 28$ & $55 / 53$ \\
\hline BMI $\left(\mathrm{kg} / \mathrm{m}^{2}\right)$ & $25.75 \pm 3.40$ & $26.09 \pm 4.28$ & $25.25 \pm 3.69$ \\
\hline Scr (mg/dL) & $0.93 \pm 0.30$ & $1.63 \pm\left. 0.3\right|^{*}$ & $1.65 \pm 0.29 *$ \\
\hline Albumin $(g / L)$ & $39.85 \pm 3.53$ & $27.13 \pm 3.76^{*}$ & $27.01 \pm 3.79 *$ \\
\hline WBC $\left(\times 10^{9} / \mathrm{L}\right)$ & $7.40 \pm 1.61$ & $13.14 \pm 6.46^{*}$ & $14.40 \pm 7.17^{*}$ \\
\hline CRP $(\mathrm{mg} / \mathrm{L})$ & $4.19 \pm 1.24$ & $70.22 \pm 24.64^{*}$ & $101.18 \pm 31.43^{* \#}$ \\
\hline РCT (mg/L) & $5.33 \pm 1.64$ & $8.04 \pm 1.53^{*}$ & $12.36 \pm 3.79$ *\# \\
\hline SOFA score & - & $0.41 \pm 0.25$ & $7.96 \pm 2.35^{\#}$ \\
\hline APACHE II score & - & - & $|4.50 \pm 4.4|^{\#}$ \\
\hline Death (n) & - & 0 & 29 (26.9\%) \\
\hline
\end{tabular}

Notes: $* P<0.05$, compared with Healthy control; ${ }^{*} P<0.05$, compared with pneumonia patients.

Abbreviations: BMI, body mass index; Scr, serum creatinine; WBC, white blood cells, CRP, C-reactive protein; PCT, procalcitonin; SOFA, sequential organ failure assessment; APACHE II, acute pathology and chronic health evaluation II.

$500 \mathrm{ng}$ isolated total RNA were retro-transcriptional to generate their complementary single-stranded cDNA through the miRNA cDNA synthesis Kit. Finally, the miRNA qPCR Assay kit, miRNA-specific primers, and template cDNA (2 mug) were mixed to perform PCR amplification on ABI-Prism 7900 sequence detection system. The relative mRNA levels of miR-486-5p were calculated as $2^{-\Delta \Delta C t}$ and U6 snRNA was used for normalization.

\section{Follow-Up Program}

All patients were followed up with appropriate treatment regimens under sepsis guidelines. The survival status of patients within 28 days was recorded, and the predictive ability of miR-486-5p levels on the survival outcome of patients was studied.

\section{Statistical Analysis}

Statistical analysis was executed by SPSS 23.0 and GraphPad Prism 6.0. Data were expressed as mean \pm SD. Spearman coefficient correlation analysis was used for correlation analysis. The diagnostic value of the ROC curve for detecting miRNA was developed by plotting the 1-specific sensitivity. Kaplan-Meier estimator and Cox regression hazards analysis of the predictive significance in 28-day survival. Student's $t$-test and one-way ANOVA analysis were used to compare the difference between groups. $P<$ 0.05 represented a statistical significance.

\section{Results}

\section{General Demographic Characteristics}

The clinical data of sepsis, pneumonia-infected, and healthy control included in the study are listed in
Table 1. There were no statistical differences in age, gender, and BMI $(P>0.05)$. However, compared with healthy controls, Scr, WBC, CRP, and PCT were remarkedly decreased in sepsis and pneumonia-infected patients, while Albumin was significantly increased $(P<0.05)$. In addition, although sepsis patients had no difference in Scr, Albumin, and WBC from pneumonia-infected patients, the CRP, PCT, and SOFA score was significantly higher than that of pneumonia-infected patients $(P<0.05)$.

\section{Serum miR-486-5p Were Elevated in Sepsis Patients}

miR-486-5p expression was then measured in enrolled patients with sepsis $(n=108)$, pneumonia-infected $(n=60)$, and healthy controls $(n=101)$. As shown in Figure 1 , miR486-5p was significantly enhanced in the two kinds of patients compared to healthy controls $(P<0.05)$. More interestingly, the serum miR-486-5p in sepsis patients was higher than that in pneumonia-infected patients $(P<0.05)$.

\section{Correlation Analysis of miR-486-5p and Key Indicators in Sepsis Patients}

To further study the specific role of serum miR-486-5p in the clinical treatment of sepsis patients, we analyzed the correlation between miR-486-5p and SOFA and APACHE II, the key clinical indicators of disease severity, and results showed that miR-486-5p was positively related with both SOFA $(r=0.603)$ and APACHE II $(r=0.556$, Figure 2$)$.

In addition, Spearman's coefficient analysis also showed that common inflammatory markers in sepsis patients, namely clinical diagnostic biomarkers PCT $(r=0.543)$ and 


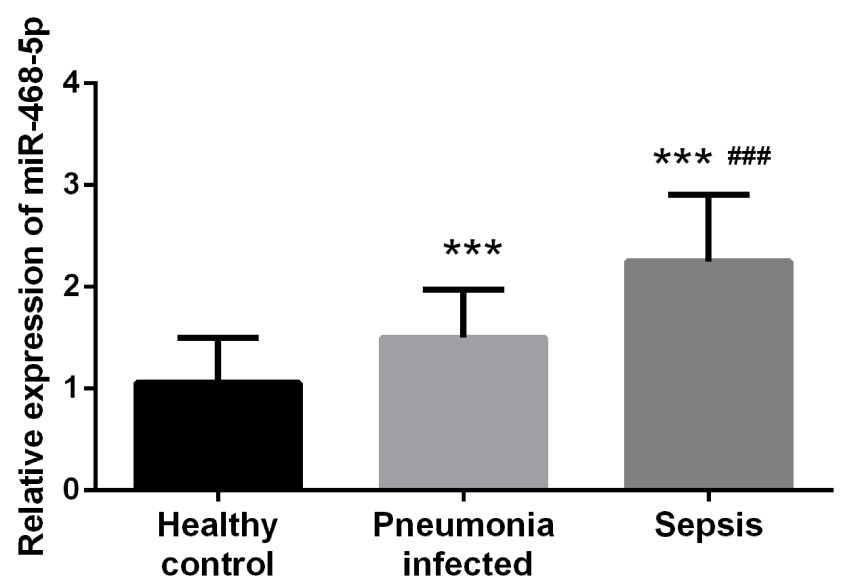

Figure I Detection of serum miR-486-5p in sepsis patients. The signature elevation of miR-486-5p in sepsis and pneumonia-infected patients compared with healthy control. And the serum miR-486-5p level of sepsis patients was higher than pneumonia-infected patients. (***P $<0.00 \mathrm{I}$, compared with healthy control; ${ }^{\# \#} p<0.00$ I, compared with pneumonia-infected patients).
CRP $(r=0.515)$ were positively correlated with serum miR486-5p. The above findings suggest that miR-486-5p is positively related to the severity of sepsis and inflammatory response, and may be a diagnostic biomarker.

The Potential Value of miR-486-5p in the Differential Diagnosis of Sepsis

Subsequently, to confirm our previous speculation that miR486-5p might play a certain role in the diagnosis of sepsis patients, we performed ROC analysis. The results showed that miR-486-5p could distinguish sepsis patients from healthy individuals, with an AUC of 0.914. And based on the cutoff value of 1.30 yielded sensitivity and specificity were $88 \%$ and $84 \%$, the positive likelihood ratio (+LR) was 5.50 , and the negative LR (-LR) was 0.143 . We then assessed

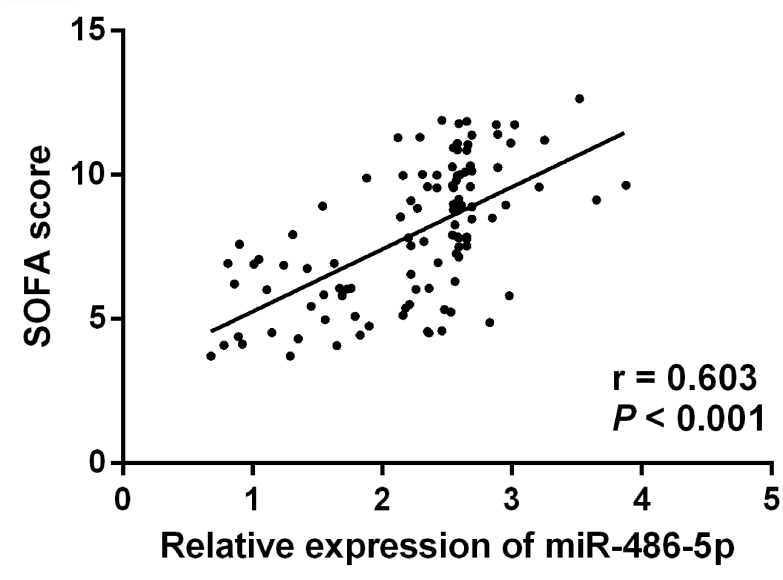

C

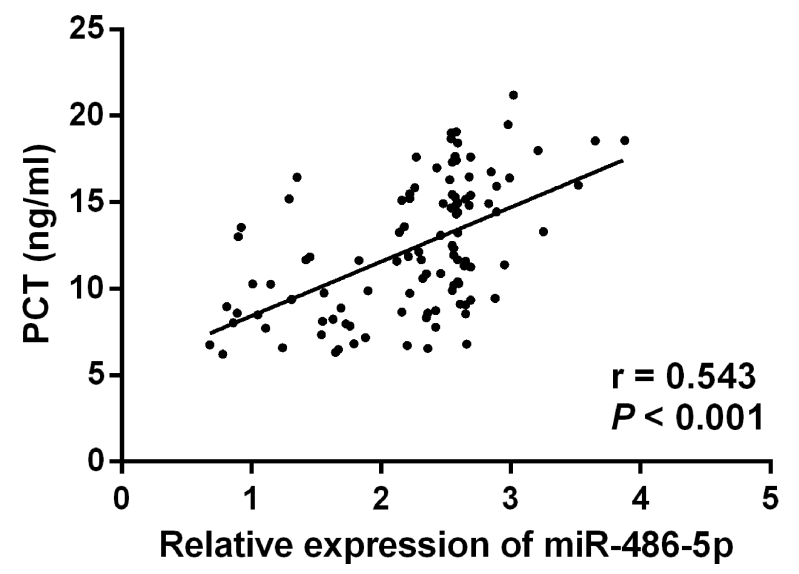

B

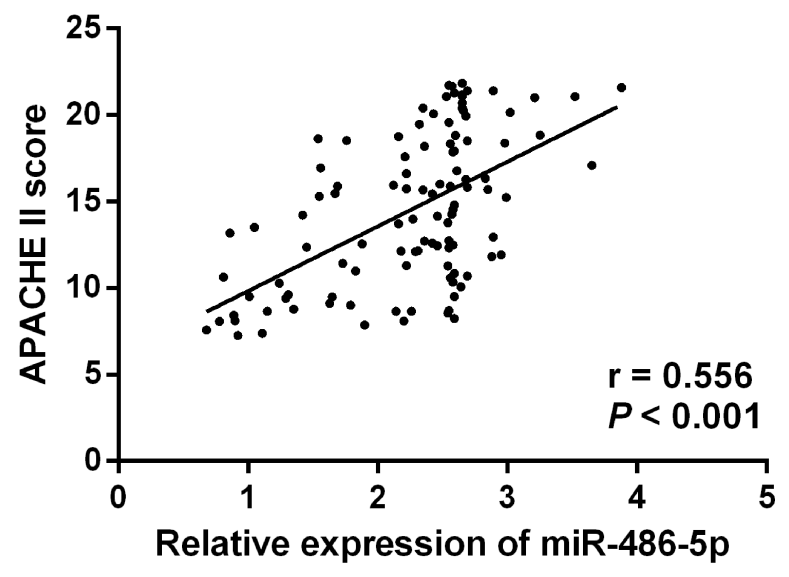

D

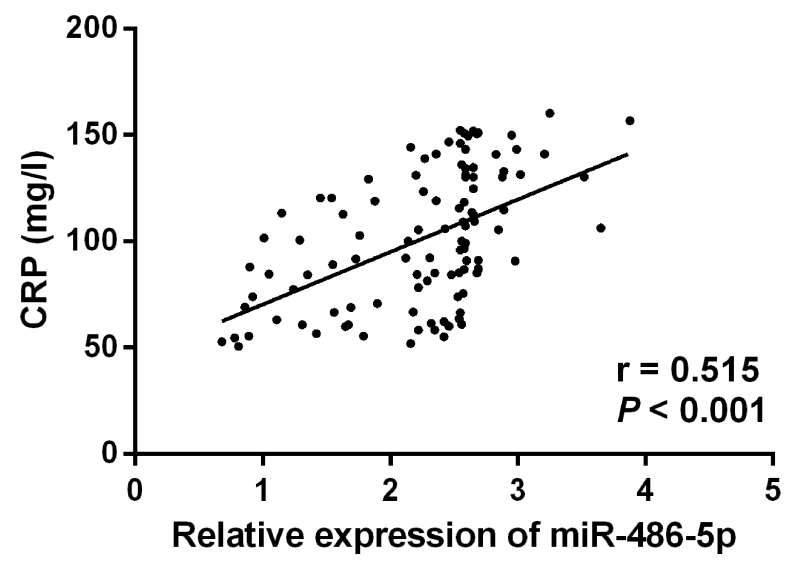

Figure 2 Correlation analysis of miR-486-5p level with disease severity and inflammatory factor in sepsis. Spearman correlation analysis demonstrated that the relative level of miR-486-5p in serum of sepsis patients was positively correlated with SOFA score (A), APACHE II (B), PCT (C), and CRP (D). 
the potential diagnostic significance of miR-486-5p in differentiating sepsis patients from pneumonia-infected. Serum miR-486-3p could distinguish sepsis from patients with pneumonia-infected. The AUC of ROC was 0.814, and with the cutoff value was 2.11 yielded the sensitivity was $72.2 \%$, specificity was $91.7 \%$, the +LR was 8.699 , and -LR was 0.303 (Figure 3). All the above have confirmed our speculation that serum miR-486-5p is a feasible biomarker for the diagnosis of sepsis.

\section{Predictive Value of miR-486-5p in 28-Day Survival}

A total of 29 patients died during the 28-day follow-up period in the present research, we sought to analyze the predictive significance of miR-486-5p in 28-day survival in sepsis patients. Firstly, according to the mean expression of miR-486-5p $(2.25 \pm 0.66)$ in the serum of sepsis patients, we divided them into high and low groups. Kaplan-Meier estimator analysis evaluated the effects of different miR-486-5p levels on 28-day survival in sepsis patients. As shown in Figure 4, the 28-day survival rate of patients with high miR-486-5p was markedly reduced than that of patients with low miR-486-5p (log-rank $P=0.012$, Figure 4). Multivariate Cox regression analysis also confirmed that miR-486-5p $(\mathrm{HR}=3.057,95 \% \mathrm{CI}=1.385$
$17.817, P=0.014$, Table 2) was an independent predictor of 28-day survival in sepsis.

\section{Discussion}

In the current study, we confirmed that miR-486-5p has a good clinical significance in sepsis. Increased miR-486$5 \mathrm{p}$ in sepsis was positively related to disease severity and inflammation biomarkers PCT and CRP. It is a viable diagnostic marker for sepsis, identifying sepsis from healthy controls or patients with pneumonia-infected. Furthermore, high serum miR-486-5p was an independent predictor of poor 28-day survival with sepsis.

MiR-486-5p is initially identified in the liver of human fetuses. ${ }^{20}$ Previous research has reported that miR-486-5p plays a crucial regulatory role as an oncogene or tumor suppressor gene in a variety of cancers. For example, miR-486-5p acts as a tumor suppressor in breast cancer, ${ }^{21}$ colorectal cancer, ${ }^{22}$ or esophageal cancer, ${ }^{23}$ and acts as an oncogene in cervical cancer. ${ }^{24}$ Several recent surveys have indicated that abnormally expressed miRNAs have the potential to serve as biomarkers for biological diagnosis, prediction, and prognosis of diseases, including sepsis. Such as, the decrease of urine miR-22-3p is a predictive marker for the occurrence of acute kidney injury and 28-day survival in sepsis patients. ${ }^{7}$ MiR-122 is elevated in sepsis and can be used as a biomarker for its early diagnosis. ${ }^{25}$ It is
A

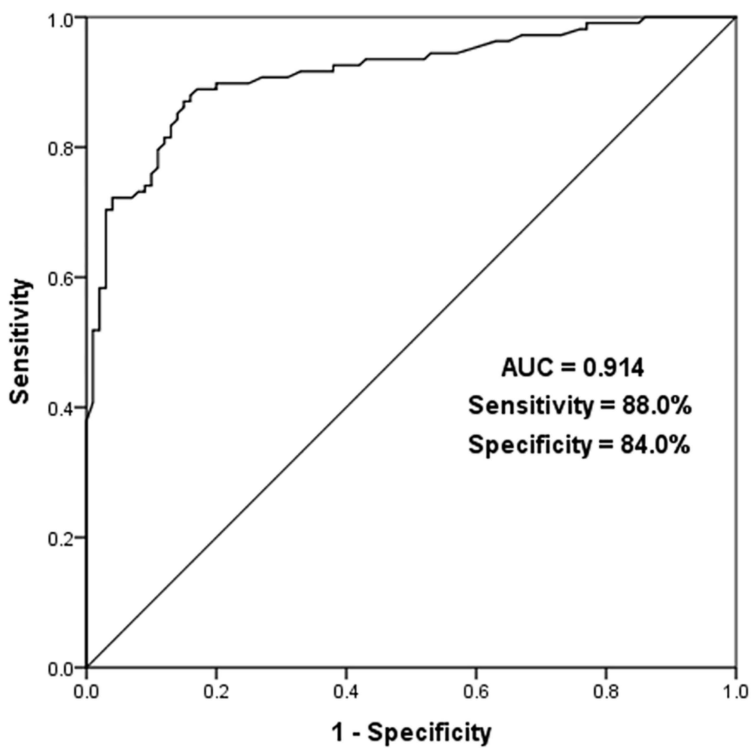

B

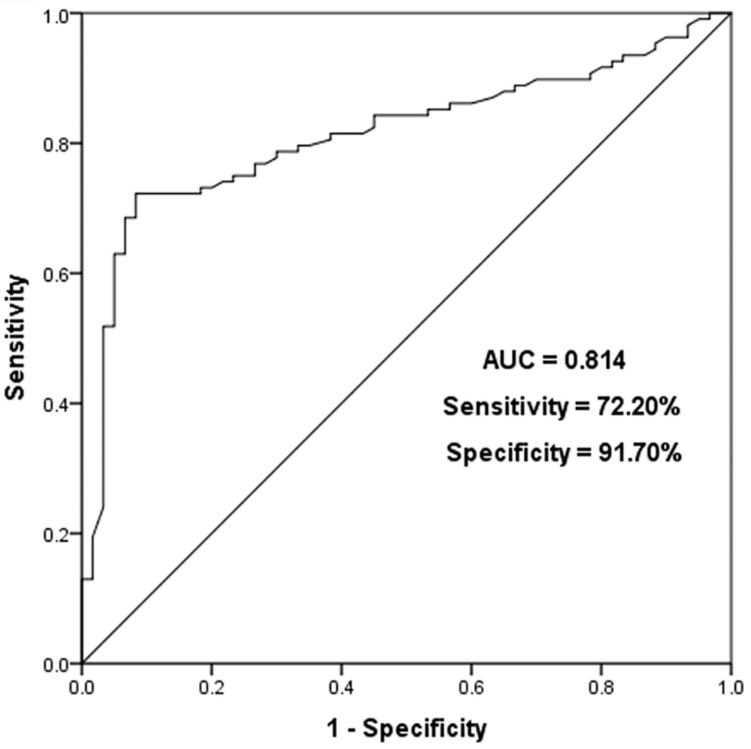

Figure 3 Diagnostic significant of miR-486-5p in sepsis patients. (A) ROC curve confirmed that serum miR-486-5p to distinguish sepsis patients from healthy controls. The AUC was 0.914 , with the cutoff value was 1.30 yielded specificity and sensitivity were $84 \%$ and $88 \%$, the positive likelihood ratio (+LR) was 5.50 , and the negative LR (-LR) was 0.143 . (B) miR-486-5p can remarkedly identify sepsis patients from pneumonia patients. AUC was 0.814 , with the cutoff value was 2 .II yielded the sensitivity was $72.2 \%$, specificity was $91.7 \%$, the +LR was 8.699 , and - LR was 0.303 . 


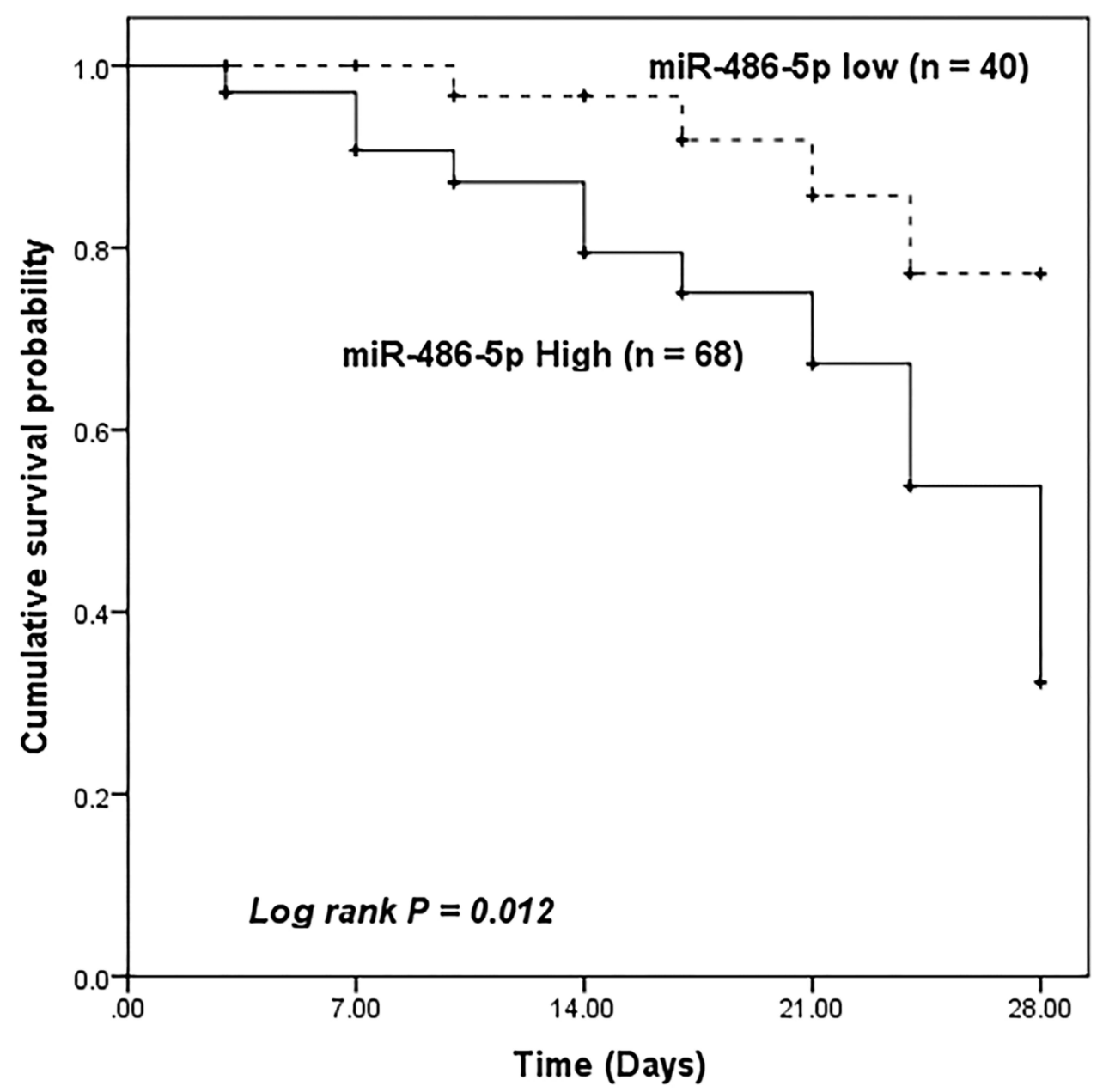

Figure 4 Kaplan-Meier estimator was used to calculate the predictive significance of miR-486-5p in 28-day survival in sepsis patients. Sepsis patients with high levels of miR486-5p were worse than those with low levels (Log-rank $P=0.012$ ).

interesting to note that recent studies have compared multiple miRNAs differentially expressed in sepsis and healthy, and miR-486-5p was found in the upregulated miRNAs. ${ }^{15}$ Moreover, the abnormal miR-486-5p is involved in a variety of inflammatory responses, such as knee arthritis, and is related to the severity of the disease. ${ }^{26}$ miR- $486-5 p$ is involved in chronic obstructive pulmonary disease by regulating the inflammatory. ${ }^{27}$ The process of sepsis involves inflammation. Sepsis patients with the infection will cause the activation of phagocytes and produce normal responses such as inflammatory mediators to facilitate the repair of injured tissue. However, sepsis can occur when inflammatory cytokines are produced uncontrolled and beyond the scope of the local infections environment and cause a broader response. ${ }^{12}$

Given the above, we speculate whether miR-486-5p plays a certain function in sepsis. First, we studied three groups of subjects who were included in the present research.
Serum miR-486-5p was elevated in patients, which was in agreement with the results of previous surveys. More importantly, we found that the serum miR-486-5p in sepsis patients was higher than that in pneumonia-infected patients. The results indicate that serum miR-486-5p may play pivotal regulators in the occurrence of sepsis.

Due to the lack of specific symptoms of sepsis and the diagnostic significance of laboratory examination is poor. ${ }^{28}$ At present, CRP and PCT, which are indicators of inflammation, are used as biomarkers for early clinical diagnosis of sepsis. However, the sensitivity and specificity of different studies are quite different. CRP and PCT are also known to be elevated in pneumonia-infected patients, so their specificity and sensitivity are limited. ${ }^{29}$ Although blood culture is the gold standard for sepsis, identification is complicated by high false-negative results. ${ }^{6}$ The APACHE II score and SOFA score are widely used to assess sepsis risk and prognosis; however, 
Table 2 Multivariate Cox Analysis for the 28-Day Survival of Sepsis

\begin{tabular}{|l|c|c|c|}
\hline \multirow{2}{*}{ Characteristics } & \multicolumn{3}{|c|}{ Multivariate Analysis } \\
\cline { 2 - 4 } & HR & $95 \%$ Cl & P \\
\hline miR-486-5p & 4.968 & $1.385-17.817$ & 0.014 \\
Age & 1.244 & $0.414-3.736$ & 0.698 \\
Gender & 1.780 & $0.729-4.349$ & 0.206 \\
BMI & 1.268 & $0.491-3.276$ & 0.624 \\
Scr & 0.419 & $0.157-1.117$ & 0.082 \\
Albumin & 1.752 & $0.760-4.040$ & 0.188 \\
WBC & 1.805 & $0.775-4.205$ & 0.171 \\
CRP & 2.764 & $0.973-7.853$ & 0.056 \\
PCT & 0.151 & $0.031-0.740$ & 0.020 \\
SOFA score & 3.122 & $1.226-7.954$ & 0.017 \\
APACHE II score & 2.823 & $1.074-7.421$ & 0.035 \\
\hline
\end{tabular}

Abbreviations: BMI, body mass index; Scr, serum creatinine; WBC, white blood cells, CRP, C-reactive protein; PCT, procalcitonin; SOFA, sequential organ failure assessment; APACHE II, acute pathology and chronic health evaluation II.

multiple measurements are required due to their poor specificity. $^{30}$ Therefore, we analyzed the correlation between serum miR-486-5p and common diagnostic criteria PCT, CRP, APACHE II, and SOFA score by Spearman coefficient analysis. The present investigation showed that miR-486-5p was positively correlated with all of them, indicating that the increased miR-486-5p was in correlation with both the inflammatory and the severity of disease in sepsis. Subsequent ROC analysis attempted to confirm the diagnostic significance of miR-486-5p in sepsis patients. The present investigation confirmed that miR486-5p could not only identify sepsis patients from healthy controls but also clearly distinguish sepsis and pneumoniainfected patients, showing a potential good diagnostic biomarker for sepsis.

Sepsis is a disease with rapid progression and high mortality in ICU, so early identification and timely intervention are very important. After determining the clinical diagnostic significance of miR-486-5p for sepsis, we attempted to further analyze the predictive significance of miR-486-5p for 28-day survival. In this study, a total of 29 patients died during the 28-day follow-up period, and most of them occurred in patients with high miR-486-5p expression. The Kaplan-Meier estimator analysis confirmed this view. And multivariate Cox analysis validated that elevated miR-486$5 \mathrm{p}$ was an independent predictor of sepsis patients.

Sirtuin 1 (SIRT1), a nicotinamide adenine dinucleotide (NAD+)-dependent deacetylase, was demonstrated to be significantly reduced in sepsis patients. ${ }^{31}$ Moreover, SIRT1 is one of the most promising molecules to relieve sepsis. It can inhibit inflammation, oxidative stress, and metabolomic abnormalities of sepsis patients by regulating different signaling pathways, and reduce sepsis's organ damage. ${ }^{32}$ Reducing the SITR1 inhibitive effect of miR-133a on LPSinduced sepsis was reversed. ${ }^{33}$ Shikonin, a traditional Chinese medicine extract, improved LPS-induced sepsis cardiac dysfunction by SIRT1. ${ }^{34}$ We predicted that SIRT1 was the target gene of miR-486-5p through the online biological software miRDB, and miR-486-5p targeting SIRT1 has been demonstrated in non-small cell lung cancer ${ }^{35}$ and myocardial ischemia-reperfusion injury. ${ }^{36}$ Therefore, we speculate that miR-486-5p may be involved in the progress of sepsis by targeting SIRT1. However, its specific molecular mechanism still needs further study.

Finally, this study also has some limitations. First of all, this study only evaluated the 28-day survival of patients, which is a short-term outcome, and a larger sample is needed for long-term outcomes. Secondly, this current study only involves clinical studies, while in vitro studies and related molecular mechanism studies will be examined. What is more, strict inclusion and exclusion criteria were implemented in this study to eliminate interference factors as much as possible. However, a larger sample is needed for further study in clinical practice.

Taking everything into account, the present investigation confirmed that miR-486-5p may be a feasible diagnostic biomarker for sepsis patients, and is positively correlated with inflammation and severity of patients, and may also predict 28-day survival of patients.

\section{Disclosure}

The authors report no conflicts of interest in this work.

\section{References}

1. Zheng X, Leung KS, Wong MH, et al. Long non-coding RNA pairs to assist in diagnosing sepsis. BMC Genomics. 2021;22(1):275. doi:10.1186/s12864-021-07576-4

2. Tang Y, Yang X, Shu H, et al. Bioinformatic analysis identifies potential biomarkers and therapeutic targets of septic-shock-associated acute kidney injury. Hereditas. 2021;158(1):1-10. doi:10.1186/s41065-02100176-y

3. Na L, Ding H, Xing E, et al. Lnc-MEG3 acts as a potential biomarker for predicting increased disease risk, systemic inflammation, disease severity, and poor prognosis of sepsis via interacting with miR-21. $J$ Clin Lab Anal. 2020;34(4):e23123. doi:10.1002/jcla.23123

4. Tuzun F, Ozkan H, Cetinkaya M, et al. Is European Medicines Agency (EMA) sepsis criteria accurate for neonatal sepsis diagnosis or do we need new criteria? PLoS One. 2019;14(6):e0218002. doi:10.1371/journal.pone. 0218002

5. Llobat L, Gourbault O. Role of MicroRNAs in human osteosarcoma: future perspectives. Biomedicines. 2021;9(5):463. doi:10.3390/ biomedicines 9050463 
6. Wang D, Han L. Downregulation of miR-1184 serves as a diagnostic biomarker in neonatal sepsis and regulates LPS-induced inflammatory response by inhibiting IL-16 in monocytes. Exp Ther Med. 2021;21:350. doi:10.3892/etm.2021.9781

7. Zhang H, Che L, Wang Y, et al. Deregulated microRNA-22-3p in patients with sepsis-induced acute kidney injury serves as a new biomarker to predict disease occurrence and 28-day survival outcomes. Int Urol Nephrol. 2021. doi:10.1007/s11255-021-02784-z

8. Liu Z, Yang D, Gao J, et al. Discovery and validation of miR-452 as an effective biomarker for acute kidney injury in sepsis. Theranostics. 2020;10(26):11963-11975. doi:10.7150/thno.50093

9. Liu HC, Han DS, Hsu CC, et al. Circulating MicroRNA-486 and MicroRNA-146a serve as potential biomarkers of sarcopenia in the older adults. BMC Geriatr. 2021;21(1):86. doi:10.1186/s12877-02102040-0

10. Zheng $\mathrm{X}, \mathrm{Xu} \mathrm{K}$, Zhu L, et al. MiR-486-5p act as a biomarker in endometrial carcinoma: promotes cell proliferation, migration, invasion by targeting MARK1. Onco Targets Ther. 2020;13:4843-4853. doi:10.2147/OTT.S246841

11. Ninawe A, Guru SA, Yadav $P$, et al. miR-486-5p: a prognostic biomarker for chronic myeloid leukemia. ACS Omega. 2021;6 (11):7711-7718. doi:10.1021/acsomega.1c00035

12. Zheng $\mathrm{C}$, Wu D, Shi $\mathrm{S}$, et al. miR-34b-5p promotes renal cell inflammation and apoptosis by inhibiting aquaporin-2 in sepsis-induced acute kidney injury. Ren Fail. 2021;43(1):291-301. doi:10.1080/0886022X.2021.1871922

13. Cirillo F, Catellani C, Lazzeroni P, et al. MiRNAs regulating insulin sensitivity are dysregulated in Polycystic Ovary Syndrome (PCOS) ovaries and are associated with markers of inflammation and insulin sensitivity. Front Endocrinol. 2019;10:879. doi:10.3389/ fendo.2019.00879

14. Luo Q, Zhu J, Zhang Q, et al. MicroRNA-486-5p promotes acute lung injury via inducing inflammation and apoptosis by targeting OTUD7B. Inflammation. 2020;43(3):975-984. doi:10.1007/s10753020-01183-3

15. Vasilescu C, Rossi S, Shimizu M, et al. MicroRNA fingerprints identify miR-150 as a plasma prognostic marker in patients with sepsis. PLoS One. 2009;4(10):e7405. doi:10.1371/journal.pone.0007405

16. Vandewalle J, Libert C. Glucocorticoids in sepsis: to be or not to be. Front Immunol. 2020;11:1318. doi:10.3389/fimmu.2020.01318

17. Shankar-Hari M, Phillips GS, Levy ML, et al. Developing a new definition and assessing new clinical criteria for septic shock: for the third international consensus definitions for sepsis and septic shock (Sepsis-3). JAMA. 2016;315(8):775-787. doi:10.1001/jama.2016.0289

18. Vincent JL, de Mendonca A, Cantraine F, et al. Use of the SOFA score to assess the incidence of organ dysfunction/failure in intensive care units: results of a multicenter, prospective study. Working group on "sepsis-related problems" of the European society of intensive care medicine. Crit Care Med. 1998;26(11):1793-1800. doi:10.1097/ 00003246-199811000-00016

19. Singer M, Deutschman CS, Seymour CW, et al. The third international consensus definitions for sepsis and septic shock (sepsis-3). JAMA. 2016;315(8):801-810. doi:10.1001/jama.2016.0287

20. Fu H, Tie Y, Xu C, et al. Identification of human fetal liver miRNAs by a novel method. FEBS Lett. 2005;579(17):3849-3854. doi:10.1016/j. febslet.2005.05.064

21. Tahiri A, Leivonen SK, Lüders $T$, et al. Deregulation of cancer-related miRNAs is a common event in both benign and malignant human breast tumors. Carcinogenesis. 2014;35(1):76-85. doi:10.1093/carcin/bgt333
22. Liu X, Chen X, Zeng K, et al. DNA-methylation-mediated silencing of miR-486-5p promotes colorectal cancer proliferation and migration through activation of PLAGL2/IGF2/beta-catenin signal pathways. Cell Death Dis. 2018;9(10):1037. doi:10.1038/s41419018-1105-9

23. Yi Y, Lu X, Chen J, et al. Downregulated miR-486-5p acts as a tumor suppressor in esophageal squamous cell carcinoma. Exp Ther Med. 2016;12(5):3411-3416. doi:10.3892/etm.2016.3783

24. Li C, Zheng X, Li W, et al. Serum miR-486-5p as a diagnostic marker in cervical cancer: with investigation of potential mechanisms. $B M C$ Cancer. 2018;18(1):61. doi:10.1186/s12885-017-3753-z

25. Abou El-Khier NT, Zaki ME, Alkasaby NM. Study of MicroRNA-122 as a diagnostic biomarker of sepsis. Egypt J Immunol. 2019;26:105-116.

26. Kong R, Gao J, Si Y, et al. Combination of circulating miR-19b-3p, miR-122-5p and miR-486-5p expressions correlates with risk and disease severity of knee osteoarthritis. Am $J$ Transl Res. 2017;9:2852-2864.

27. Zhang J, Xu Z, Kong L, et al. miRNA-486-5p promotes COPD progression by targeting HAT1 to regulate the TLR4-triggered inflammatory response of alveolar macrophages. Int $J$ Chron Obstruct Pulmon Dis. 2020;15:2991-3001. doi:10.2147/COPD. $\mathrm{S} 280614$

28. Lopez R, Vial C, Graf J, et al. Platelet count in patients with mild disease at admission is associated with progression to severe hantavirus cardiopulmonary syndrome. Viruses. 2019;11:693. doi:10.3390/ v11080693

29. Guo S, Mao X, Liang M. The moderate predictive value of serial serum CRP and PCT levels for the prognosis of hospitalized community-acquired pneumonia. Respir Res. 2018;19:193. doi:10.1186/s12931-018-0877-x

30. Zhao D, Li S, Cui J, et al. Plasma miR-125a and miR-125b in sepsis: correlation with disease risk, inflammation, severity, and prognosis. $J$ Clin Lab Anal. 2020;34(2):e23036. doi:10.1002/jcla.23036

31. Ling H, Li Q, Duan ZP, et al. LncRNA GAS5 inhibits miR-579-3p to activate SIRT1/PGC-1alpha/Nrf2 signaling pathway to reduce cell pyroptosis in sepsis-associated renal injury. Am J Physiol Cell Physiol. 2021;321(1):C117-C133. doi:10.1152/ajpcell.00394.2020

32. Li L, Liu M, Cao M, et al. Research progress on SIRT1 and sepsis. Histol Histopathol. 2019;34:1205-1215.

33. Chen L, Xie W, Wang L, et al. MiRNA-133a aggravates inflammatory responses in sepsis by targeting SIRT1. Int Immunopharmacol. 2020;88:106848. doi:10.1016/j.intimp.2020.106848

34. Guo T, Jiang ZB, Tong ZY, et al. Shikonin ameliorates LPS-induced cardiac dysfunction by SIRT1-dependent inhibition of NLRP3 inflammasome. Front Physiol. 2020;11:570441. doi:10.3389/ fphys. 2020.570441

35. Gao ZJ, Yuan WD, Yuan JQ, et al. miR-486-5p functions as an oncogene by targeting PTEN in non-small cell lung cancer. Pathol Res Pract. 2018;214(5):700-705. doi:10.1016/j.prp.2018.03.013

36. Sun XH, Wang X, Zhang Y, et al. Exosomes of bone-marrow stromal cells inhibit cardiomyocyte apoptosis under ischemic and hypoxic conditions via miR-486-5p targeting the PTEN/PI3K/AKT signaling pathway. Thromb Res. 2019;177:23-32. doi:10.1016/j. thromres.2019.02.002 


\section{Publish your work in this journal}

The Journal of Inflammation Research is an international, peerreviewed open-access journal that welcomes laboratory and clinical findings on the molecular basis, cell biology and pharmacology of inflammation including original research, reviews, symposium reports, hypothesis formation and commentaries on: acute/chronic inflammation; mediators of inflammation; cellular processes; molecular mechanisms; pharmacology and novel anti-inflammatory drugs; clinical conditions involving inflammation. The manuscript management system is completely online and includes a very quick and fair peerreview system. Visit http://www.dovepress.com/testimonials.php to read real quotes from published authors.

Submit your manuscript here: https://www.dovepress.com/journal-of-inflammation-research-journal 